\title{
Characterizations of woven frames
}

\author{
A. Bhandari ${ }^{\mathrm{a}}$, S. Mukherjee $\mathrm{a}^{\mathrm{a}, 1, *}$ \\ ${ }^{a}$ Dept. of Mathematics, NIT Meghalaya, Shillong 793003, India
}

\begin{abstract}
In a separable Hilbert space $\mathcal{H}$, two frames $\left\{f_{i}\right\}_{i \in I}$ and $\left\{g_{i}\right\}_{i \in I}$ are said to be woven if there are constants $0<A \leq B$ so that for every $\sigma \subset I$, $\left\{f_{i}\right\}_{i \in \sigma} \cup\left\{g_{i}\right\}_{i \in \sigma^{c}}$ forms a frame for $\mathcal{H}$ with the universal bounds $A, B$. This article provides methods of constructing woven frames. In particular, bounded linear operators are used to create woven frames from a given frame. Several examples are discussed to validate the results. Moreover, the notion of woven frame sequences is introduced and characterized.

Keywords: Frames, Woven Frames, Gap, Angle

2010 MSC: Primary 42C15; Secondary 46C07, 97H60
\end{abstract}

\section{Introduction}

Hilbert space frame was first initiated by D. Gabor [1] in 1946 to reconstruct signals using fourier co-efficients. Later, in 1986, frame theory was reintroduced and popularized by Daubechies, Grossman and Meyer [2].

Frame theory literature became richer through several generalizations, namely, $G$-frame (generalized frames) [3], $K$-frame (frames for operators

\footnotetext{
${ }^{*}$ Corresponding author

Email addresses: animesh@nitm.ac.in (A. Bhandari), saikat.mukherjee@nitm.ac.in (S. Mukherjee)

${ }^{1}$ Supported by DST-SERB project MTR/2017/000797.
} 
(atomic systems)) [4], fusion frame (frames of subspaces) ([ㅁ, 6] $]$ ), $K$-fusion frame (atomic subspaces) 7], etc. and some spin-off applications by means of Gabor analysis in ([8, 9]), dynamical system in mathematical physics in [10], nature of shift invariant spaces on the Heisenberg group in [11], characterizations of discrete wavelet frames in $\mathbb{C}^{\mathbb{N}}$ in [12], extensions of dual wavelet frames in [13], constructions of disc wavelets in [14], orthogonality of frames on locally compact abelian groups in [15] and many more.

Let us consider a scenario: suppose in a sensor network system, there are sensors $A_{1}, A_{2}, \cdots, A_{n}$ which capture data to produce certain results. These sensors can be characterized by frames. In case one of these sensors, say $A_{k}$, fails to operate due to some technical reason, then the results obtained from these sensors may contain errors. Now assume that there are another set of sensors $B_{1}, B_{2}, \cdots, B_{n}$ which does play similar role as $A_{i}$ 's. In addition, in the case of $A_{k}$ fails, $B_{k}$ can substitute so that obtained results are error free. Such an intertwinedness between two sets of sensors, or in general between two frames, leads to the idea of weaving frames. Weaving frames or woven frames were recently introduced by Bemrose et. al. in [16]. After that Deepshikha et. al. produced a generalized form of weaving frames in [17], they also studied the weaving properties of generalized continuous frames in [18], vector-valued (super) weaving frames in [19].

This article focuses on study, characterize and explore several properties of woven frames. The outline of this article is organized as follows. Section 2 is devoted to the basic definitions and results related to various kinds of frames, angle and gap between subspaces. Moreover, the characterizations of woven frames are analyzed in Section 3. Finally, woven frame sequences are established in Section 4.

Throughout the paper, $\mathcal{H}$ is a separable Hilbert space. We denote by 
$\mathcal{H}^{n}$ an $n$-dimensinal Hilbert space, $\left\{e_{i}\right\}_{i \in[n]}, e_{i}=\left(\delta_{i, k}\right)_{k \geq 1}$ is an orthonormal basis in $\mathcal{H}^{n}, \mathcal{L}\left(\mathcal{H}^{n}\right)$ to be a collection of all bounded, linear operators on $\mathcal{H}^{n}$, $R(T)$ is denoted as the range of the operator $T$, by $\hat{\delta}(M, N)$ we denote the gap between two closed subspaces $M$ and $N$ of a Hilbert space $\mathcal{H}, c_{0}(M, N)$ is denoted as the cosine of the minimal angle between $M$ and $N,[n]=$ $\{1,2, \cdots, n\}$ and the index set $I$ is either finite or countably infinite. Given $J \subset I$, a Bessel sequence $\left\{f_{i}\right\}_{i \in I}$ and $\left\{c_{i}\right\} \in l^{2}(I)$, we define $T_{J_{f}}\left(\left\{c_{i}\right\}\right)=$ $\sum_{i \in J} c_{i} f_{i}$. It is to be noted that $T_{J_{f}}$ is well-defined.

\section{Preliminaries}

In this section we recall basic definitions and results needed in this paper. For more details we refer the books written by Casazza and Kutyniok [20] and Ole Christensen [21].

\subsection{Frame}

A collection $\left\{f_{i}\right\}_{i \in I}$ in $\mathcal{H}$ is called a frame for $\mathcal{H}$ if there exist constants $A, B>0$ such that

$$
A\|f\|^{2} \leq \sum_{i \in I}\left|\left\langle f, f_{i}\right\rangle\right|^{2} \leq B\|f\|^{2},
$$

for all $f \in \mathcal{H}$. The numbers $A, B$ are called frame bounds. The operator $S: \mathcal{H} \rightarrow \mathcal{H}$, defined by $S f=\sum_{i \in I}\left\langle f, f_{i}\right\rangle f_{i}$ is called the frame operator for $\left\{f_{i}\right\}_{i \in I}$. It is well-known that the frame operator is linear, bounded, positive, self-adjoint and invertible.

Definition 2.1. Let $\left\{f_{i}\right\}_{i \in I}$ and $\left\{g_{i}\right\}_{i \in I}$ be two frames for $\mathcal{H}$. If for all $f \in \mathcal{H}, f=\sum_{i \in I}\left\langle f, g_{i}\right\rangle f_{i}$, then $\left\{g_{i}\right\}_{i \in I}$ is called a dual frame of $\left\{f_{i}\right\}_{i \in I}$. If $S$ is the frame operator of $\left\{f_{i}\right\}_{i \in I}$, then $\left\{S^{-1} f_{i}\right\}_{i \in I}$ is said to be the canonical dual frame of $\left\{f_{i}\right\}_{i \in I}$. 
Proposition 2.2. ([21, 22] ]) A finite family $\left\{f_{i}\right\}_{i \in[m]}$ in $\mathcal{H}^{n}$, forms a frame for $\mathcal{H}^{n}$ if and only if $\operatorname{span}\left\{f_{i}\right\}_{i \in[m]}=\mathcal{H}^{n}$.

\subsection{Woven and full spark frame}

In a Hilbert space $\mathcal{H}$, a family of frames $\left\{f_{i j}\right\}_{i \in \mathbb{N}, j \in[M]}$ is said to be weakly woven if for any partition $\left\{\sigma_{j}\right\}_{j \in[M]}$ of $\mathbb{N},\left\{f_{i j}\right\}_{i \in \sigma_{j}, j \in[M]}$ forms a frame for $\mathcal{H}$.

Also, in $\mathcal{H}$, two frames $\mathcal{F}=\left\{f_{i}\right\}_{i \in I}$ and $\mathcal{G}=\left\{g_{i}\right\}_{i \in I}$ are said to be woven if for every $\sigma \subseteq I,\left\{f_{i}\right\}_{i \in \sigma} \cup\left\{g_{i}\right\}_{i \in \sigma^{c}}$ also forms a frame for $\mathcal{H}$ and the associated frame operator for every weaving is defined as,

$$
S_{\mathcal{F} \mathcal{G}} f=\sum_{i \in \sigma}\left\langle f, f_{i}\right\rangle f_{i}+\sum_{i \in \sigma^{c}}\left\langle f, g_{i}\right\rangle g_{i}, \text { for all } f \in \mathcal{H}
$$

Theorem 2.3. [16] In $\mathcal{H}$, two frames are weakly woven if and only if they are woven.

Moreover, a frame with $m$ elements in $\mathcal{H}^{n}$, is said to be a full spark frame if every subset of the frame, with cardinality $n$, is also a frame for $\mathcal{H}^{n}$. For example, if $\left\{e_{i}\right\}_{i \in[2]}, e_{i}=\left(\delta_{i, k}\right)_{k \geq 1}$, is an orthonormal basis in $\mathbb{R}^{2}$, then $\left\{e_{1}, e_{2}, e_{1}+e_{2}\right\}$ is a full spark frame for $\mathbb{R}^{2}$. Furthermore, if every element of a finite frame can be represented as a linear combination of the remaining others, then the frame is called a weak full spark frame.

For example, if $\left\{e_{i}\right\}_{i \in[3]}$ is an orthonormal basis of $\mathbb{R}^{3},\left\{e_{1}, e_{1}, e_{2}, e_{2}, e_{3}, e_{3}\right\}$ is a weak full spark frame but not a full spark frame. In this context, it is a fortuitous evident that every nontrivial (other than exact) full spark frame is also a weak full spark frame.

Proposition 2.4. [16] Let $\left\{f_{i j}\right\}_{i \in I}$ be a collection of Bessel sequences in $\mathcal{H}$ with bounds $B_{j}$ 's for every $j \in[m]$, then every weaving forms a Bessel 
sequence with bound $\sum_{j \in[m]} B_{j}$ and norm of corresponding synthesis operator is bounded by $\sqrt{\sum_{j \in[m]} B_{j}}$.

Proposition 2.5. Every frame sequence is a Bessel sequence.

Proof. Let $\left\{f_{i}\right\}_{i \in I}$ be a frame sequence in $\mathcal{H}$, then it forms a frame for $F=\overline{\operatorname{span}}\left\{f_{i}\right\}_{i \in I}$. Therefore $\mathcal{H}=F \oplus F^{\perp}$ and hence for every $f \in \mathcal{H}$ we have $f=f_{F}+f_{F^{\perp}}$. Therefore for some $B>0$ we obtain,

$$
\sum_{i \in I}\left|\left\langle f, f_{i}\right\rangle\right|^{2}=\sum_{i \in I}\left|\left\langle f_{F}, f_{i}\right\rangle\right|^{2} \leq B\left\|f_{F}\right\|^{2} \leq B\|f\|^{2}
$$

Remark 2.6. The converse implication of the above proposition is not necessarily true, which is evident from the following fact:

Consider $\left\{e_{i}\right\}_{i \in \mathbb{N}}$ as an orthonormal basis for $\mathcal{H}$ and let us define

$$
f_{i}=e_{i}+e_{i+1}, i \in \mathbb{N} \text {. }
$$

Then $\left\{f_{i}\right\}_{i \in \mathbb{N}}$ forms a Bessel sequence in $\mathcal{H}$ but not a frame sequence. For detail discussion regarding the same we refer the Example 5.1.10 in [21].

Lemma 2.7. Let $\left\{f_{i}\right\}_{i \in I}$ and $\left\{g_{i}\right\}_{i \in I}$ be frames for $\mathcal{H}$ with bounds $A_{1}, B_{1}$ and $A_{2}, B_{2}$, respectively. Then the following results are equivalent:

1. $\left\{f_{i}\right\}_{i \in I}$ and $\left\{g_{i}\right\}_{i \in I}$ are woven.

2. For every $\sigma \subset I$, if $S_{\sigma}$ is the associated frame operator for the corresponding weaving, then for every $f \in \mathcal{H}$ we have $\left\|S_{\sigma} f\right\| \geq k\|f\|$ for some $k>0$, independent of $\sigma$.

Proof. $\underline{(1 \Longrightarrow 2)}$ 
Let $\left\{f_{i}\right\}_{i \in I}$ and $\left\{g_{i}\right\}_{i \in I}$ be woven with universal frame bounds $A, B$. Therefore for every $\sigma \subset I$ and for every $f \in \mathcal{H}$ we have,

$$
A\|f\|^{2} \leq \sum_{i \in \sigma}\left|\left\langle f, f_{i}\right\rangle\right|^{2}+\sum_{i \in \sigma^{c}}\left|\left\langle f, g_{i}\right\rangle\right|^{2} \leq B\|f\|^{2} .
$$

If $S_{\sigma}$ is the associated frame operator for the corresponding weaving, then from the above inequality we have,

$$
A\|f\|^{2} \leq\left\langle S_{\sigma} f, f\right\rangle \leq B\|f\|^{2} .
$$

Therefore,

$$
\left\|S_{\sigma} f\right\|=\sup _{\|g\|=1}\left|\left\langle S_{\sigma} f, g\right\rangle\right| \geq\left\langle S_{\sigma} f, \frac{f}{\|f\|}\right\rangle \geq A\|f\| .
$$

$\underline{(2 \Longrightarrow 1)}$

For every $f \in \mathcal{H}$ and $\sigma \subset I, S_{\sigma} f=T_{\sigma} T_{\sigma}^{*} f$, where $T_{\sigma}, T_{\sigma}^{*}$ are the corresponding synthesis and analysis operators, respectively; and we have $k^{2}\|f\|^{2} \leq\left\|S_{\sigma} f\right\|^{2}=\left\|T_{\sigma} T_{\sigma}^{*} f\right\| \leq\left\|T_{\sigma}\right\|^{2}\left\|T_{\sigma}^{*} f\right\|^{2}$ and hence we obtain,

$$
\frac{k^{2}}{B_{1}+B_{2}}\|f\|^{2} \leq\left\|T_{\sigma}^{*} f\right\|^{2}=\sum_{i \in \sigma}\left|\left\langle f, f_{i}\right\rangle\right|^{2}+\sum_{i \in \sigma^{c}}\left|\left\langle f, g_{i}\right\rangle\right|^{2} .
$$

The universal upper frame bound for the weaving will be achieved by Proposition 2.4 .

Theorem 2.8. [16] In $\mathcal{H}^{n}$, two frames $\left\{f_{i}\right\}_{i \in[m]}$ and $\left\{g_{i}\right\}_{i \in[m]}$ are woven if and only if for every $\sigma \subseteq[m], \operatorname{span}\left(\left\{f_{i}\right\}_{i \in \sigma} \cup\left\{g_{i}\right\}_{i \in \sigma^{c}}\right)=\mathcal{H}^{n}$.

\subsection{Gap and angle between subspaces}

Let $M$ and $N$ be two closed subspaces of a Hilbert space $\mathcal{H}$. Then the gap between $M$ and $N$ is given by, $\hat{\delta}(M, N)=\max \{\delta(M, N), \delta(N, M)\}$, where $\delta(M, N)=\sup _{x \in S_{M}} \operatorname{dist}(x, N), S_{M}$ is the unit sphere in $M$ and $\operatorname{dist}(x, N)$ is the distance from $x$ to $N$. 
Again the cosine of the angle between two closed subspaces $M$ and $N$ of a Hilbert space $\mathcal{H}$ is given by,

$c(M, N)=\sup \left\{|\langle x, y\rangle|: x \in M \cap(M \cap N)^{\perp},\|x\| \leq 1, y \in N \cap(M \cap N)^{\perp},\|y\| \leq 1\right\}$ and the cosine of the minimal angle of the same is given by,

$$
c_{0}(M, N)=\sup \{|\langle x, y\rangle|: x \in M,\|x\| \leq 1, y \in N,\|y\| \leq 1\}
$$

For the extensive discussion regarding the gap and the angle between two subspaces, we refer $([23,24,25])$.

Remark 2.9. [25] Let $M$ and $N$ be two closed subspaces of a Hilbert space $\mathcal{H}$. Then the followings are satisfied:

1. $\delta(M, N)=0$ if and only if $M \subset N$.

2. $\hat{\delta}(M, N)=0$ if and only if $M=N$.

Lemma 2.10. [24] Let $M$ and $N$ be two closed subspaces of a Hilbert space $\mathcal{H}$. Then $c_{0}(M, N)=0$ if and only if $M \perp N$.

Theorem 2.11. [24] Let $M$ and $N$ be two closed subspaces of a Hilbert space $\mathcal{H}$. Then the following statements are equivalent:

1. $c_{0}(M, N)<1$.

2. $M \cap N=\{0\}$ and $M+N$ is closed.

Theorem 2.12. (Douglas' factorization theorem [26]) Let $\mathcal{H}_{1}, \mathcal{H}_{2}$, and $\mathcal{H}$ be Hilbert spaces and $S \in \mathcal{L}\left(\mathcal{H}_{1}, \mathcal{H}\right), T \in \mathcal{L}\left(\mathcal{H}_{2}, \mathcal{H}\right)$. Then the following results are equivalent:

1. $R(S) \subseteq R(T)$.

2. $S S^{*} \leq \alpha T T^{*}$ for some $\alpha>0$.

3. $S=T L$ for some $L \in \mathcal{L}\left(\mathcal{H}_{1}, \mathcal{H}_{2}\right)$. 


\section{Characterization of Woven Frames}

In this section, we characterize woven frames, mainly through constructions of frames from given frames. The proposed constructions are based on the images of a given frame by means of bounded linear operators. Before diving into the main results, we start the discussion with the following Proposition.

Proposition 3.1. Let $\left\{f_{i}\right\}_{i \in[m]}$ be a frame for $\mathcal{H}^{n}$. Suppose $f_{m+1}=0$, then $\left\{\left(f_{i}-f_{i+1}\right)\right\}_{i \in[m]}$ is also a frame for $\mathcal{H}^{n}$ and these two frames are woven.

Proof. The proof will be followed from elementary row operations.

Remark 3.2. In the above Proposition instead of $f_{m+1}=0$, if $f_{m+1}=f_{1}$, then $\left\{\left(f_{i}-f_{i+1}\right)\right\}_{i \in[m]}$ may not be a frame for $\mathcal{H}^{n}$. For example, let $\left\{e_{i}\right\}_{i \in[3]}$ be an orthonormal basis in $\mathbb{R}^{3}$, then $\left\{-e_{1}+e_{2}, e_{1}+e_{2},-2 e_{1}+e_{2}-e_{3}, e_{1}+\right.$ $\left.e_{2}+e_{3}\right\}$ is a frame for $\mathbb{R}^{3}$. But clearly, $\left\{\left(f_{i}-f_{i+1}\right)\right\}_{i \in[4]}=\left\{-2 e_{1}, 3 e_{1}+\right.$ $\left.e_{3},-3 e_{1}-2 e_{3}, 2 e_{1}+e_{3}\right\}$ is not a frame for $\mathbb{R}^{3}$.

But if this is a frame, then they must be woven, which is evident from the fact that $f=\sum_{i \in[m]} b_{i}\left(f_{i}-f_{i+1}\right)$ can be written as $f=\left(b_{1}-b_{j}\right) f_{1}+\left(b_{2}-\right.$ $\left.b_{1}\right) f_{2}+\ldots\left(b_{j}-b_{j-1}\right) f_{j}+\left(b_{j+1}-b_{j}\right)\left(f_{j+1}-f_{j+2}\right)+\ldots\left(b_{m-1}-b_{j}\right)\left(f_{m-1}-\right.$ $\left.f_{m}\right)+\left(b_{m}-b_{j}\right)\left(f_{m}-f_{m+1}\right)$.

Remark 3.3. If $\left\{f_{i}\right\}_{i \in[m]}$ is a frame for $\mathcal{H}^{n}$ and suppose $f_{m+1}=0$, then $\left\{\left(\alpha f_{i}+\beta f_{i+1}\right)\right\}_{i \in[m]}, \alpha, \beta \neq 0$, is also a frame for $\mathcal{H}^{n}$ and they are woven.

In the following result, we present conditions under which image of a given frame under an idempotent operator is woven with the said frame. 
Lemma 3.4. Let $F(\neq 0) \in \mathcal{L}(\mathcal{H})$ be a closed range, idempotent operator with $R(F)=R\left(F^{*}\right)$. Suppose $\left\{f_{i}\right\}_{i \in I}$ is a frame for $R\left(F^{*}\right)$, then $\left\{F f_{i}\right\}_{i \in I}$ is also a frame for $R\left(F^{*}\right)$ and they are woven.

Proof. Let $\left\{f_{i}\right\}_{i \in I}$ be a frame for $R\left(F^{*}\right)$ with bounds $A, B$. Then for every $f \in R\left(F^{*}\right)$ we have,

$$
\sum_{i \in I}\left|\left\langle f, F f_{i}\right\rangle\right|^{2}=\sum_{i \in I}\left|\left\langle F^{*} f, f_{i}\right\rangle\right|^{2} \leq B\left\|F^{*} f\right\|^{2} \leq B\|F\|^{2}\|f\|^{2}
$$

Again since $f \in R\left(F^{*}\right)=R(F),\|f\|^{2}=\left\|\left(F^{*}\right)^{\dagger} F^{*} f\right\|^{2} \leq\left\|\left(F^{*}\right)^{\dagger}\right\|^{2}\left\|F^{*} f\right\|^{2}$ and hence $\frac{\|f\|^{2}}{\left\|\left(F^{*}\right)^{\dagger}\right\|^{2}} \leq\left\|F^{*} f\right\|^{2}$. Therefore for every $f \in R\left(F^{*}\right)$ we obtain,

$$
\sum_{i \in I}\left|\left\langle f, F f_{i}\right\rangle\right|^{2}=\sum_{i \in I}\left|\left\langle F^{*} f, f_{i}\right\rangle\right|^{2} \geq A\left\|F^{*} f\right\|^{2} \geq \frac{A}{\left\|\left(F^{*}\right)^{\dagger}\right\|^{2}}\|f\|^{2} .
$$

Consequently, $\left\{F f_{i}\right\}_{i \in I}$ forms a frame for $R\left(F^{*}\right)$.

Moreover, for every $f \in R\left(F^{*}\right)$, there exists $g \in \mathcal{H}$ such that $F^{*} g=f$ and since $F^{2}=F$, for every $\sigma \subset I$ and for all $f \in R\left(F^{*}\right)$ we obtain,

$$
\begin{aligned}
\sum_{i \in \sigma}\left|\left\langle f, f_{i}\right\rangle\right|^{2}+\sum_{i \in \sigma^{c}}\left|\left\langle f, F f_{i}\right\rangle\right|^{2} & =\sum_{i \in \sigma}\left|\left\langle F^{*} g, f_{i}\right\rangle\right|^{2}+\sum_{i \in \sigma^{c}}\left|\left\langle F^{*} g, F f_{i}\right\rangle\right|^{2} \\
& =\sum_{i \in \sigma}\left|\left\langle g, F f_{i}\right\rangle\right|^{2}+\sum_{i \in \sigma^{c}}\left|\left\langle g, F f_{i}\right\rangle\right|^{2} \\
& =\sum_{i \in I}\left|\left\langle g, F f_{i}\right\rangle\right|^{2} \\
& =\sum_{i \in I}\left|\left\langle f, f_{i}\right\rangle\right|^{2} .
\end{aligned}
$$

Therefore, our assertion is tenable.

Remark 3.5. It is to be noted that, if one of the conditions of $F^{2}=F$ and $R(F)=R\left(F^{*}\right)$ fails, then the conclusion of the above Lemma may not hold. This is evident from the following two examples. 
Example 3.6. Consider an idempotent operator $F$ on $\mathbb{R}^{2}$ so that $F e_{1}=$ $e_{1}+2 e_{2}, F e_{2}=0$, then $R(F)=\operatorname{span}\left\{e_{1}+2 e_{2}\right\} \neq \operatorname{span}\left\{e_{1}\right\}=R\left(F^{*}\right)$. Now for the frame $\mathcal{F}=\left\{e_{1}, e_{2}, e_{1}+e_{2}\right\}$ for $\mathbb{R}^{2}, F(\mathcal{F})=\left\{e_{1}+2 e_{2}, 0, e_{1}+2 e_{2}\right\}$ is not a frame for $R\left(F^{*}\right)$.

Example 3.7. Consider an operator $F$ on $\mathbb{R}^{3}$ so that $F e_{1}=e_{1}+e_{2}, F e_{2}=$ $-e_{1}+e_{2}, F e_{3}=0$, then $F^{2} \neq F$ but $R(F)=R\left(F^{*}\right)$. Now let us choose a frame $\left\{f_{i}\right\}_{i \in[3]}=\left\{e_{1}, e_{1}-e_{2}, 2 e_{1}\right\}$ for $R\left(F^{*}\right)$, then $\left\{F f_{i}\right\}_{i \in[3]}=\left\{e_{1}+\right.$ $\left.e_{2}, 2 e_{1}, 2 e_{1}+2 e_{2}\right\}$ is also a frame for $R\left(F^{*}\right)$, but they are not woven, which can be verified for $\sigma=\{1,3\}$.

In the following outcomes we study woven-ness of frames and their images under invertible operators.

Remark 3.8. The image of a frame under invertible operators is not necessarily woven with the frame, which is evident from the following example: $\mathcal{F}=\left\{e_{2}, e_{1}+e_{2}, 2 e_{2}\right\}$ is a frame for $\mathbb{R}^{2}$. Let us consider an invertible operator $T$ so that $T e_{1}=e_{1}-e_{2}, T e_{2}=-e_{1}-e_{2}$. Then $T \mathcal{F}=\left\{-\left(e_{1}+\right.\right.$ $\left.\left.e_{2}\right),-2 e_{2},-2\left(e_{1}+e_{2}\right)\right\}$ is also a frame for $\mathbb{R}^{2}$, however they are not woven, which can be verified by considering $\sigma=\{1,3\}$.

Proposition 3.9. The image of woven frames under invertible operator preserves their woven-ness.

Proof. Let $\left\{f_{i}\right\}_{i \in I}$ and $\left\{g_{i}\right\}_{i \in I}$ be woven in $\mathcal{H}$ with universal bounds $A, B$ and suppose $T \in \mathcal{L}(\mathcal{H})$ is an invertible operator. Then $\left\{T f_{i}\right\}_{i \in I}$ and $\left\{T g_{i}\right\}_{i \in I}$ are also frames for $\mathcal{H}$. 
For every $\sigma \subset I$ and for every $f \in \mathcal{H}$ we obtain,

$$
\begin{aligned}
\sum_{i \in \sigma}\left|\left\langle f, T f_{i}\right\rangle\right|^{2}+\sum_{i \in \sigma^{c}}\left|\left\langle f, T g_{i}\right\rangle\right|^{2} & =\sum_{i \in \sigma}\left|\left\langle T^{*} f, f_{i}\right\rangle\right|^{2}+\sum_{i \in \sigma^{c}}\left|\left\langle T^{*} f, g_{i}\right\rangle\right|^{2} \\
& \geq A\left\|T^{*} f\right\|^{2} \\
& \geq \frac{A}{\left\|T^{-1}\right\|^{2}}\|f\|^{2}
\end{aligned}
$$

The upper frame bound of the respective weaving will be achieved from the Proposition 2.4.

In the following theorem we discuss a necessary and sufficient condition of woven frames.

Theorem 3.10. Let $\mathcal{F}=\left\{f_{i}\right\}_{i \in I}$ and $\mathcal{G}=\left\{g_{i}\right\}_{i \in I}$ be two frames for $\mathcal{H}$. Then they are woven if and only if $R\left(T_{\mathcal{F G}}\right)=\mathcal{H}$, where $T_{\mathcal{F G}}$ is the associated synthesis operator of the respective weaving.

Proof. Since $\mathcal{F}$ and $\mathcal{G}$ are frames for $\mathcal{H}$, by Proposition 2.4 every weaving is a Bessel sequence and hence $T_{\mathcal{F G}}$ is well-defined.

Let $R\left(T_{\mathcal{F G}}\right)=\mathcal{H}=R\left(I_{\mathcal{H}}\right)$. Therefore using Theorem 2.12, there exists $A>0$ and for every $f \in \mathcal{H}$ we have $\left\|T_{\mathcal{F} \mathcal{G}}^{*} f\right\|^{2} \geq A\|f\|^{2}$ and hence for every $\sigma \subset I$ we obtain,

$$
\sum_{i \in \sigma}\left|\left\langle f, f_{i}\right\rangle\right|^{2}+\sum_{i \in \sigma^{c}}\left|\left\langle f, g_{i}\right\rangle\right|^{2} \geq A\|f\|^{2}
$$

The converse implication will be followed from the definition of woven frame.

Example 3.11. $\mathcal{F}=\left\{e_{1}+e_{2}, e_{1}+2 e_{2}, e_{1}-e_{2}\right\}$ is a frame for $\mathbb{R}^{2}$. If $S$ is its frame operator, then $S \mathcal{F}=\left\{5 e_{1}+8 e_{2}, 7 e_{1}+14 e_{2}, e_{1}-4 e_{2}\right\}$. It is to be noted that the associated synthesis operators of every weaving are onto. 
Since invertible operators preserve linear independency of vectors, so it is a natural intuition that a finite frame is woven with its image under the associated frame operator.

Problem 1: If $\left\{f_{i}\right\}_{i \in I}$ is a frame for $\mathcal{H}$ with the associated frame operator $S$, Can $\left\{f_{i}\right\}_{i \in I}$ and $\left\{S f_{i}\right\}_{i \in I}$ woven?

At this moment, we are impotent to deliver an affirmative response, although we strongly believe that the same can be executed in this context.

If so, then using Proposition [3.9, it is evident that $\left\{f_{i}\right\}_{i \in I}$ and $\left\{S^{-1} f_{i}\right\}_{i \in I}$ are woven.

Problem 2: Whether a frame is woven with its dual?

\section{Woven Frame Sequence}

A family $\left\{f_{i}\right\}_{i \in I}$ in $\mathcal{H}$ is said to be a frame sequence if it forms a frame for its closed, linear span. It is to be noted that $\left\{f_{i}\right\}_{i \in I}$ is not necessarily a frame for $\mathcal{H}$. In this section we explore the possibilities of two frame sequences together, through the concept of woven frames, form a frame for $\mathcal{H}$.

Definition 4.1. Two frame sequences $\mathcal{F}=\left\{f_{i}\right\}_{i \in I}$ and $\mathcal{G}=\left\{g_{i}\right\}_{i \in I}$ in $\mathcal{H}$, are said to be woven frame sequences, if for every $\sigma \subset I,\left\{f_{i}\right\}_{i \in \sigma} \cup$ $\left\{g_{i}\right\}_{i \in \sigma^{c}}$ forms a frame for $\mathcal{H}$.

Example 4.2. For example, In $\mathbb{R}^{3}$, the frame sequences $\left\{e_{1}+2 e_{3}, e_{1}-\right.$ $\left.e_{3},-e_{1}+2 e_{3}, e_{1}+3 e_{3}\right\}$ and $\left\{e_{1}-e_{2}, e_{1}+2 e_{2},-e_{1}+3 e_{2}, e_{1}-2 e_{2}\right\}$ are woven whereas $\left\{e_{1}+2 e_{3}, e_{1}-e_{3},-e_{1}+2 e_{3}, e_{1}+3 e_{3}\right\}$ and $\left\{e_{1}-e_{2}, e_{1}+2 e_{2},-e_{1}+\right.$ $\left.3 e_{2}, e_{1}\right\}$ are not.

The notion of woven frame sequences is beneficial for its practical importance, because instead of two given frames, if we consider two frame 
sequences, then less restriction is there in our primary assumption and due to this fact, it is cost-effective.

Theorem 4.3. Let $\mathcal{F}=\left\{f_{i}\right\}_{i \in[m]}$ and $\mathcal{G}=\left\{g_{i}\right\}_{i \in[m]}$ be two frame sequences in $\mathcal{H}^{n}$. Then the following statements are satisfied:

1. $\mathcal{F}$ and $\mathcal{G}$ are not woven if there exists a non-trivial $\sigma \subset[m]$ so that $c_{0}\left\{\operatorname{span}\left(\mathcal{F}_{\sigma} \cup \mathcal{G}_{\sigma^{c}}\right), \operatorname{span}\left(\mathcal{F}_{\sigma^{c}} \cup \mathcal{G}_{\sigma}\right)\right\}<1$.

2. If for every non-trivial $\sigma \subset[m], \hat{\delta}\left\{\operatorname{span}\left(\mathcal{F}_{\sigma} \cup \mathcal{G}_{\sigma^{c}}\right), \operatorname{span}\left(\mathcal{F}_{\sigma^{c}} \cup \mathcal{G}_{\sigma}\right)\right\}=0$ and $c_{0}\left\{\left(\operatorname{span}\left(\mathcal{F}_{\sigma} \cup \mathcal{G}_{\sigma^{c}}\right)\right),\left(\operatorname{span}\left(\mathcal{F}_{\sigma^{c}} \cup \mathcal{G}_{\sigma}\right)\right)^{c}\right\}=0=c_{0}\left\{\left(\operatorname{span}\left(\mathcal{F}_{\sigma^{c}} \cup\right.\right.\right.$ $\left.\left.\mathcal{G}_{\sigma}\right)\right),\left(\operatorname{span}\left(\mathcal{F}_{\sigma} \cup \mathcal{G}_{\sigma^{c}}\right)\right)^{c}$, then $\mathcal{F}$ and $\mathcal{G}$ are woven.

PROOF. Using Lemma 2.10 and Theorem 2.11, our assertions are quickly plausible.

Theorem 4.4. In $\mathcal{H}^{n}$, if $\mathcal{F}=\left\{f_{i}\right\}_{i \in[m]}$ and $\mathcal{G}=\left\{g_{i}\right\}_{i \in[m]}$ are two woven frame sequences, then for every non-trivial $\sigma \subset[m]$,

$$
\hat{\delta}\left\{\operatorname{span}\left(\mathcal{F}_{\sigma} \cup \mathcal{G}_{\sigma^{c}}\right), \operatorname{span}\left(\mathcal{F}_{\sigma^{c}} \cup \mathcal{G}_{\sigma}\right)\right\}=0
$$

Proof. If $\mathcal{F}$ and $\mathcal{G}$ are woven, then for every non-trivial $\sigma \subset[m]$, both $\mathcal{F}_{\sigma} \cup \mathcal{G}_{\sigma^{c}}$ and $\mathcal{F}_{\sigma^{c}} \cup \mathcal{G}_{\sigma}$ constitute frames for $\mathcal{H}^{n}$. Hence the conclusion directly follows from the Remark 2.9 .

Remark 4.5. It is to be noted that, the two foregoing outcomes also hold for characterizing woven frames.

In the following results we explore sufficient conditions for woven-ness between frame and frame sequence. The following theorem shows that wovenness is preserved under perturbation. 
Theorem 4.6. Let $\mathcal{F}=\left\{f_{i}\right\}_{i \in I}, \mathcal{G}=\left\{g_{i}\right\}_{i \in I}$ be two woven frames for $\mathcal{H}$ with the universal frame bounds $A, B$ and $T_{\mathcal{G}}$ be the corresponding synthesis operator of $\mathcal{G}$. If $H=\left\{h_{i}\right\}_{i \in I}$ is a frame sequence in $\mathcal{H}$ with the associated synthesis operator $T_{H}$ so that $\left(\left\|T_{\mathcal{G}}\right\|+\left\|T_{H}\right\|\right)\left\|T_{\mathcal{G}}-T_{H}\right\|<A$, then $\mathcal{F}$ and $H$ are woven.

Proof. For every $\sigma \subset I$, let $P_{\sigma}$ be the orthogonal projection on $\overline{\operatorname{span}}\left\{e_{i}\right\}_{i \in \sigma}$ and therefore $T_{\sigma_{f}}=T_{\mathcal{F}} P_{\sigma}$.

Since $\mathcal{F}$ and $\mathcal{G}$ are woven with the universal bounds $A, B$, then using Lemma 2.7, for every $\sigma \subset I$ and every $f \in \mathcal{H}$ we have,

$$
A\|f\| \leq\left\|\sum_{i \in \sigma}\left\langle f, f_{i}\right\rangle f_{i}+\sum_{i \in \sigma^{c}}\left\langle f, g_{i}\right\rangle g_{i}\right\| .
$$

The proof will be completed with the following steps.

Step 1: For all $f \in \mathcal{H}$ and $\sigma \subset I$,

$$
\left\|\sum_{i \in \sigma^{c}}\left\langle f, g_{i}\right\rangle g_{i}-\sum_{i \in \sigma^{c}}\left\langle f, h_{i}\right\rangle h_{i}\right\| \leq\left(\left\|T_{\mathcal{G}}\right\|+\left\|T_{H}\right\|\right)\left\|T_{\mathcal{G}}-T_{H}\right\|\|f\| .
$$

proof of Step 1: Using Proposition 2.5 and utilizing the properties of the respective synthesis operators, for every $f \in \mathcal{H}$ and $\sigma \subset I$ we have,

$$
\begin{aligned}
\left\|\sum_{i \in \sigma^{c}}\left\langle f, g_{i}\right\rangle g_{i}-\sum_{i \in \sigma^{c}}\left\langle f, h_{i}\right\rangle h_{i}\right\| & \leq\left\|T_{\mathcal{G}}\right\|\left\|T_{\mathcal{G}}^{*}-T_{H}^{*}\right\|\|f\|+\left\|T_{\mathcal{G}}-T_{H}\right\|\left\|T_{H}^{*}\right\|\|f\| \\
& =\left(\left\|T_{\mathcal{G}}\right\|+\left\|T_{H}\right\|\right)\left\|T_{\mathcal{G}}-T_{H}\right\|\|f\| .
\end{aligned}
$$

Step 2: For every weaving, universal lower frame bound is $\frac{\left[A-\left\|T_{\mathcal{G}}-T_{H}\right\|\left(\left\|T_{\mathcal{G}}\right\|+\left\|T_{H}\right\|\right)\right]^{2}}{B+B_{1}}$, where $B_{1}$ is an upper frame bound for $H$.

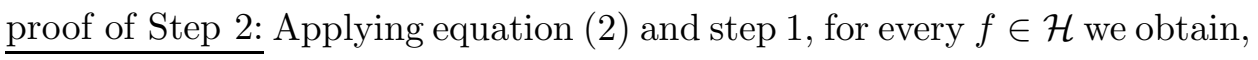

$$
\begin{aligned}
& \left\|\sum_{i \in \sigma}\left\langle f, f_{i}\right\rangle f_{i}+\sum_{i \in \sigma^{c}}\left\langle f, h_{i}\right\rangle h_{i}\right\| \\
\geq & \left\|\sum_{i \in \sigma}\left\langle f, f_{i}\right\rangle f_{i}+\sum_{i \in \sigma^{c}}\left\langle f, g_{i}\right\rangle g_{i}\right\|-\left\|\sum_{i \in \sigma^{c}}\left\langle f, g_{i}\right\rangle g_{i}-\sum_{i \in \sigma^{c}}\left\langle f, h_{i}\right\rangle h_{i}\right\| \\
\geq & {\left[A-\left\|T_{\mathcal{G}}-T_{H}\right\|\left(\left\|T_{\mathcal{G}}\right\|+\left\|T_{H}\right\|\right)\right]\|f\| . }
\end{aligned}
$$


Therefore the conclusion follows from Lemma 2.7

The universal upper frame bound of the weaving will be achieved from the Proposition 2.4.

Remark 4.7. If the frames $\mathcal{F}, \mathcal{G}$ are woven in $\mathcal{H}$ and $\mathcal{G}, H$ are woven in $\mathcal{H}$, then $\mathcal{F}$ and $H$ are not necessarily woven in $\mathcal{H}$, which is evident from the following example.

Example 4.8. Let $\mathcal{F}=\left\{e_{1}, e_{2}, 2 e_{1}\right\}, \mathcal{G}=\left\{2 e_{1},-e_{2},-2 e_{2}\right\}$ and $H=\left\{e_{1},-e_{1}, 2 e_{2}\right\}$. Then $\mathcal{F}$ and $\mathcal{G}$ are woven as well as $\mathcal{G}$ and $H$ are woven, but $\mathcal{F}$ and $H$ are not woven in $\mathbb{R}^{2}$, as if we consider $\sigma=\{3\}$ then the associated weaving is $\left\{e_{1},-e_{1}, 2 e_{1}\right\}$.

Corollary 4.9. Let $\mathcal{F}=\left\{f_{i}\right\}_{i \in I}$ be a frame for $\mathcal{H}$ with lower frame bound $A$ and $\mathcal{G}=\left\{g_{i}\right\}_{i \in I}$ be a frame sequence in $\mathcal{H}$. Let $T_{\mathcal{F}}$ and $T_{\mathcal{G}}$ be corresponding synthesis operators, respectively. Then $\mathcal{F}$ and $\mathcal{G}$ are woven if

$$
\left(\left\|T_{\mathcal{F}}-T_{\mathcal{G}}\right\|\right)\left(\left\|T_{\mathcal{F}}\right\|+\left\|T_{\mathcal{G}}\right\|\right)<A .
$$

Theorem 4.10. Let $\mathcal{F}=\left\{f_{i}\right\}_{i \in I}$ be a frame for $\mathcal{H}$ with frame bounds $A_{1}, B_{1}$ and $\mathcal{G}=\left\{g_{i}\right\}_{i \in I}$ be a frame sequence in $\mathcal{H}$ with bounds $A_{2}, B_{2}$. Suppose $0<\left(\sum_{i \in I}\left\|f_{i}\right\|^{2}\right)^{\frac{1}{2}}=\lambda_{1}<1$ and $0<\left(\sum_{i \in I}\left\|g_{i}\right\|^{2}\right)^{\frac{1}{2}}=\lambda_{2}<1$ so that $\left(\lambda_{1} \sqrt{B_{1}}+\right.$ $\left.\lambda_{2} \sqrt{B}_{2}\right)<A_{1}$. Then $\mathcal{F}$ and $\mathcal{G}$ are woven.

Proof. The proof will be completed with the following steps.

Step 1: For every $\sigma \subset I$ and every $f \in \mathcal{H}$,

$$
\left\|\sum_{i \in \sigma^{c}}\left\langle f, f_{i}\right\rangle f_{i}-\sum_{i \in \sigma^{c}}\left\langle f, g_{i}\right\rangle g_{i}\right\| \leq\left(\lambda_{1} \sqrt{B}_{1}+\lambda_{2} \sqrt{B}_{2}\right)\|f\| .
$$




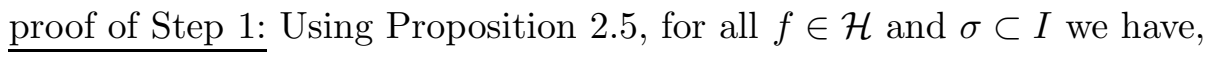

$$
\begin{aligned}
\left\|\sum_{i \in \sigma^{c}}\left\langle f, f_{i}\right\rangle f_{i}-\sum_{i \in \sigma^{c}}\left\langle f, g_{i}\right\rangle g_{i}\right\| & \leq\left\|\sum_{i \in \sigma^{c}}\left\langle f, f_{i}\right\rangle f_{i}\right\|+\left\|\sum_{i \in \sigma^{c}}\left\langle f, g_{i}\right\rangle g_{i}\right\| \\
& \leq \sum_{i \in \sigma^{c}}\left\|\left\langle f, f_{i}\right\rangle f_{i}\right\|+\sum_{i \in \sigma^{c}}\left\|\left\langle f, g_{i}\right\rangle g_{i}\right\| \\
& \leq \sum_{i \in I}\left\|\left\langle f, f_{i}\right\rangle f_{i}\right\|+\sum_{i \in I}\left\|\left\langle f, g_{i}\right\rangle g_{i}\right\| \\
& \leq\left(\sum_{i \in I}\left|\left\langle f, f_{i}\right\rangle\right|^{2}\right)^{\frac{1}{2}}\left(\sum_{i \in I}\left\|f_{i}\right\|^{2}\right)^{\frac{1}{2}} \\
& +\left(\sum_{i \in I}\left|\left\langle f, g_{i}\right\rangle\right|^{2}\right)^{\frac{1}{2}}\left(\sum_{i \in I}\left\|g_{i}\right\|^{2}\right)^{\frac{1}{2}} \\
& \leq\left(\lambda_{1} \sqrt{B}_{1}+\lambda_{2} \sqrt{B_{2}}\right)\|f\| .
\end{aligned}
$$

Step 2: For every weaving the lower frame bound is $\frac{\left[A_{1}-\left(\lambda_{1} \sqrt{B_{1}}+\lambda_{2} \sqrt{B_{2}}\right)\right]^{2}}{B_{1}+B_{2}}$. proof of Step 2: Applying Step 1, for every $f \in \mathcal{H}$ we obtain,

$$
\begin{aligned}
\left\|\sum_{i \in \sigma}\left\langle f, f_{i}\right\rangle f_{i}+\sum_{i \in \sigma^{c}}\left\langle f, g_{i}\right\rangle g_{i}\right\| & \geq\left\|\sum_{i \in I}\left\langle f, f_{i}\right\rangle f_{i}\right\|-\left\|\sum_{i \in \sigma^{c}}\left\langle f, f_{i}\right\rangle f_{i}-\sum_{i \in \sigma^{c}}\left\langle f, g_{i}\right\rangle g_{i}\right\| \\
& \geq\left[A_{1}-\left(\lambda_{1} \sqrt{B}_{1}+\lambda_{2} \sqrt{B_{2}}\right]\|f\| .\right.
\end{aligned}
$$

Therefore applying Lemma 2.7, our goal is executed.

Furthermore, universal upper frame bound of the weaving will be accomplished by utilizing the Proposition 2.4 .

\section{Acknowledgments}

The first author is highly indebted to the fiscal support of MHRD, Government of India. He also extends his massive gratitude to Dr. Manideepa Saha (NIT Meghalaya, India) and Professor Kallol Paul (Jadavpur University, India) for their useful suggestions and comments to improve this article. The second author is supported by DST-SERB project MTR/2017/000797. 


\section{References}

[1] D. Gabor, Theory of communication, J.I.E.E. 93 (1946) 429-459.

[2] I. Daubechies, A. Grossmann, Y. Mayer, Painless nonorthogonal expansions, Journal of Mathematical Physics 27 (5) (1986) 1271-1283.

[3] W. Sun, G-frames and G-riesz bases, J. Math. Anal. Appl. 322 (1) (2006) 437-452.

[4] L. Găvruţa, Frames for operators, Applied and Computational Harmonic Analysis 32 (1) (2012) 139-144.

[5] P. Casazza, G. Kutyniok, Frames of subspaces, Contemporary Math, AMS 345 (2004) 87-114.

[6] A. Khosravi, M. S. Asgari, Frames of subspaces and approximation of the inverse frame operator, Houston Journal of Mathematics 33 (3) (2007) 907-920.

[7] A. Bhandari, S. Mukherjee, Atomic subspaces for operators, Submitted, arXiv:1705.06042.

[8] T. C. Easwaran Nambudiri, K. Parthasarathy, Generalised WeylHeisenberg frame operators, Bulletin des Sciences Mathématiques 136 (1) (2012) 44-53.

[9] T. C. Easwaran Nambudiri, K. Parthasarathy, A characterisation of WeylHeisenberg frame operators, Bulletin des Sciences Mathématiques 137 (3) (2013) 322-324.

[10] S. Mishra, S. Chakraborty, Dynamical system analysis of quintom dark energy model, The European Physical Journal C 78:917. 
[11] S. Arati, R. Radha, Frames and riesz bases for shift invariant spaces on the abstract Heisenberg group, Indagationes Mathematicae 30 (1) (2019) 106-127.

[12] Deepshikha, L. Vashisht, Necessary and sufficient conditions for discrete wavelet frames in $\mathbb{C}^{\mathbb{N}}$, Journal of Geometry and Physics 117 (2017) 134 $-143$.

[13] T. C. Easwaran Nambudiri, K. Parthasarathy, Bessel sequences, wavelet frames, duals and extensions, Indagationes Mathematicae 29 (3) (2018) 907-915.

[14] L. D. Abreu, J. E. Gilbert, Wavelet-type frames for an interval, Expositiones Mathematicae 32 (3) (2014) 274-283.

[15] A. Gamber, N. K. Shukla, Pairwise orthogonal frames generated by regular representations of lca groups, Bulletin des Sciences Mathématiques 152 (2019) 40-60.

[16] T. Bemrose, P. Casazza, K. Gröchenig, M. Lammers, R. Lynch, Weaving frames, Operators and Matrices 10 (4) (2016) 1093-1116.

[17] Deepshikha, L. Vashisht, G. Verma, Generalized weaving frames for operators in Hilbert spaces, Results in Mathematics 72 (3) (2017) 1369 $-1391$.

[18] L. Vashisht, Deepshikha, Weaving properties of generalized continuous frames generated by an iterated function system, Journal of Geometry and Physics 110 (2016) $282-295$.

[19] Deepshikha, L. Vashisht, Vector-valued (super) weaving frames, Journal of Geometry and Physics 134 (2018) 48 - 57. 
[20] P. Casazza, G. Kutyniok, Finite Frames: Theory and Applications, Applied and Numerical Harmonic Analysis, Birkhäuser Boston, 2012.

[21] O. Christensen, Frames and Bases-An Introductory Course, Birkhäuser, Boston, 2008.

[22] D. Han, K. Kornelson, D. R. Larson, E. Weber, Frames for Undergraduates, AMS, 2007.

[23] O. M. Baksalary, G. Trenkler, On angles and distances between subspaces, Linear Algebra and its Applications 431 (2009) 2243-2260.

[24] F. Deutsch, The angle between subspaces of a Hilbert space, Approximation Theory, Wavelets and Applications 454 (1995) 107 - 130.

[25] T. Kato, Perturbation Theory for Linear Operators, Springer, New York, 1980.

[26] R. G. Douglas, On majorization, factorization and range inclusion of operators on Hilbert space, Proc. Amer Math. Society 17 (2) (1966) $413-415$. 